\title{
A Self-Calibrating Instrument for Measuring Conductance at Radio Frequencies
}

\author{
Leslie E. Huntley* \\ (January 12, 1965)
}

\begin{abstract}
Accuracies obtainable in measuring resistance (conductance) at radio frequencies have lagged behind those obtainable in measuring reactance (susceptance), because frequencydependent changes in the values of resistors are not as well known as are such changes in capacitors, and because no satisfactory method has existed for comparing resistors to capacitors at radio frequencies. An instrument based on the twin $T$ null circuit can be made self-calibrating at a given frequency, allowing conductances in the proper range to be measured directly in terms of a change in capacitance, without any need for accurate knowledge of the values of the circuit elements. Analysis of a practical circuit shows that such an instrument is potentially capable of measuring conductance to within a few parts in $10^{6}$ at frequencies below about $15 \mathrm{MHz}$, and that accuracies actually obtained will be limited only by the accuracy to which changes in effective capacitance are known. Conductances between 0.01 and 0.0001 mho were measured at $10^{7}$ radians/sec with an estimated error of 0.05 percent, which is the error associated with the measurement of changes in effective capacitance at that frequency.
\end{abstract}

\section{Introduction}

It has long been known that capacitors are superior to either inductors or resistors for use as impedance standards at high frequencies, because changes in their effective values with increasing frequency can be more accurately and more easily evaluated than can such changes in either resistors or inductors. For this reason, the High Frequency Impedance Group in the Electronic Calibration Center at NBS-Boulder, has decided to refer all its measurements of impedance at high frequencies to standards of capacitance [1]. ${ }^{1}$ While either null or resonance techniques can be used to compare capacitors and inductors to derived standards of capacitance, no satisfactory technique has existed for comparing resistors to capacitors at radio frequencies. The existing null techniques for measuring resistance require prior knowledge of the effective values of at least one resistor at the frequency at which the measurement is to be made. The resonance techniques, while theoretically capable of measuring resistance directly in terms of a change in capacitance, are at present capable of high accuracy only when measuring very high or very low values of resistance. Because of the lack of a satisfactory measurement technique, the accuracies obtainable in resistance measurements have lagged behind those obtainable in the measurement of reactance.

An investigation of the twin $T$ null circuit has shown that it is possible to construct an instrument capable of measuring resistance directly in terms of a change of capacitance, without prior knowledge of the values of any of the circuit elements.

This paper describes an instrument which was constructed for this purpose, and presents results which indicate that resistances, in the proper range, can be measured to an accuracy which is limited only by the accuracy to which the change in capacitance is known.

\section{Circuit Equations}

\subsection{Ideal Case (No Residual Impedances)}

The circuit to be considered (fig. 1) is adapted from one which was described by Tuttle [2], and applied to a commercial instrument by Sinclair [3], in 1940. The circuit is essentially the one which Woods [4] used in his VHF Dual Admittance Bridge in 1950. Tuttle has shown

*Radio Standards Engineering Division, NBS Boulder Laboratories, Boulder, Colo.

1 Figures in brackets indicate the lit erature references at the end of this paper. 


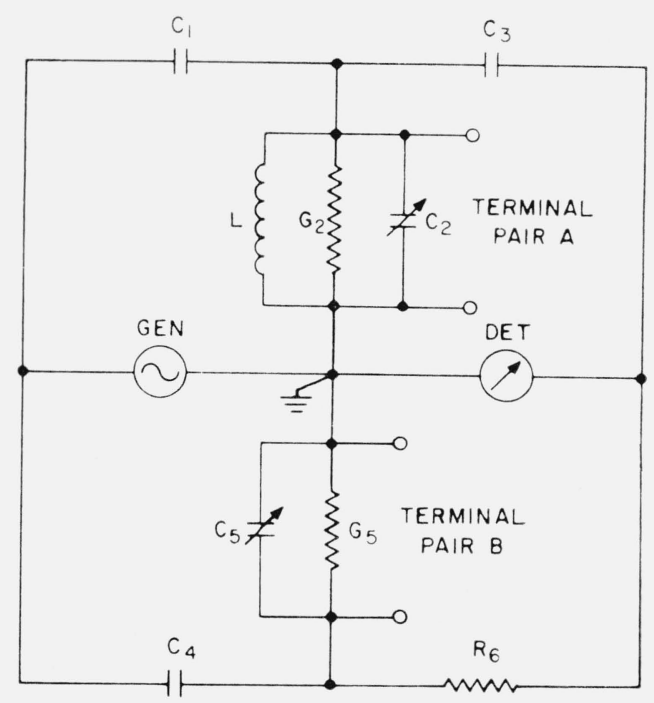

FIgURE 1. Twin $\mathrm{T}$ circuit using pure components.

that the condition for zero transmission through the twin $T$ (null condition) is given by

$$
Z_{1}+Z_{3}+\frac{Z_{1} Z_{3}}{Z_{2}}+Z_{4}+Z_{6}+\frac{Z_{4} Z_{6}}{Z_{5}}=0
$$

In the present case, $Z_{1}=\frac{1}{j \omega c_{1}}, Z_{3}=\frac{1}{j \omega c_{3}}$, etc., and the real and limaginary parts of (1) give the two null equations:

and

$$
C_{1}+C_{2}+C_{3}+\frac{C_{1} C_{3}}{C_{4}}\left(1+R_{6} G_{5}\right)=\frac{1}{\omega^{2} L}
$$

$$
\omega^{2} R_{6} C_{1} C_{3}\left(1+\frac{C_{5}}{C_{4}}\right)=G_{2}
$$

As in the Woods Bridge, a measurement can be made by connecting an unknown admittance $Y_{x}=G_{x}+j \omega C_{x}$, either to terminal-pair $A$, or to terminal-pair $B$. When $Y_{x}$ is connected to terminal-pair $A, C_{2}$ must be replaced by $C_{2}^{\prime}+C_{x}, G_{2}$ by $G_{2}^{\prime}+G_{x}$, and $C_{5}$ by $C_{5}^{\prime}$, in the null equations. Similar changes (indicated by double primes) must be made when $Y_{x}$ is connected to terminal-pair $B$. Subtracting the initial null equations from the final ones yields the four expressions

$$
\begin{gathered}
C_{x A}=-\Delta C_{2} \\
C_{x B}=-\Delta C_{5}^{\prime} \\
G_{x A}=\omega^{2}\left(R_{6} \frac{C_{1} C_{3}}{C_{4}^{\prime}}\right) \Delta C_{5} \\
G_{x B}=\frac{-\Delta C_{2}^{\prime}}{\left(R_{6} \frac{C_{1} C_{3}}{C_{4}}\right)},
\end{gathered}
$$

where

$$
\begin{array}{ll}
\Delta C_{2}=C_{2}^{\prime}-C_{2} & \Delta C_{2}^{\prime}=C_{2}^{\prime \prime}-C_{2} \\
\Delta C_{5}=C_{5}^{\prime}-C_{5} & \Delta C_{5}^{\prime}=C_{5}^{\prime \prime}-C_{5},
\end{array}
$$


and subscripts $A$ and $B$ denote the terminal-pair to which $Y_{x}$ is connected.

If $P$ is defined as

$$
P \equiv R_{6} \frac{C_{1} C_{3}}{C_{4}}
$$

then

and

$$
G_{x A}=\omega^{2} P \Delta C_{5}
$$

$$
G_{x B}=\frac{-1}{P} \Delta C_{2}^{\prime}
$$

If a conductance is measured first on terminal-pair $A$, then on terminal-pair $B$,

and

$$
G_{x A}=G_{x B}
$$

$$
\begin{aligned}
\omega^{2} P \Delta C_{5} & =-\frac{1}{P} \Delta C_{2}^{\prime} \\
P^{2} & =-\frac{1}{\omega^{2}} \frac{\Delta C_{2}^{\prime}}{\Delta C_{5}} .
\end{aligned}
$$

If $P$ is adjustable so that it can be made equal to $\frac{1}{\omega}$,

and

$$
\frac{1}{\omega^{2}}=\frac{-1}{\omega^{2}} \frac{\Delta C_{2}^{\prime}}{\Delta C_{5}}
$$

$$
\Delta C_{5}=-\Delta C_{2}^{\prime}
$$

If $P$ is adjusted to make the same change in capacitance necessary to null the instrument when $G_{x}$ is measured on terminal-pair $A$, as when it is measured on terminal-pair $B$, then $P=\frac{1}{\omega}$, and

$$
\begin{gathered}
G_{x A}=\omega \Delta C_{5} \\
G_{x B}=-\omega \Delta C_{2}^{\prime} .
\end{gathered}
$$

While $P$ has not been eliminated from the calculation of $G_{x}$, it is possible to evaluate it with great accuracy, allowing conductance to be measured with accuracies limited only by the accuracy to which a change in capacitance is known.

It should be mentioned that a conductance may be measured in terms of a change in capacitance without making $P$ equal to $1 / \omega$. If $G_{x A}=G_{x B}=G_{x}$, multiplying eq (8) by eq (9) yields the expression

$$
G_{x}^{2}=-\omega^{2} \Delta C_{2}{ }^{\prime} \Delta C_{5}
$$

and $P$ is eliminated from the calculation of $G_{x}$. Because the number of measurements needed to determine $G_{x}$ is doubled, and because accuracy decreases as the value of $P$ departs from $1 / \omega$, this method is less effective than the other, especially when more than a few measurements are to be made at a given frequency.

\subsection{Realistic Circuit (Including Residual Impedances)}

In general, residual impedances cause the effective values of the circuit elements to change with frequency and cause losses in the fixed capacitors, variable losses in the variable capacitors, and some reactance to be associated with the fixed resistors. 


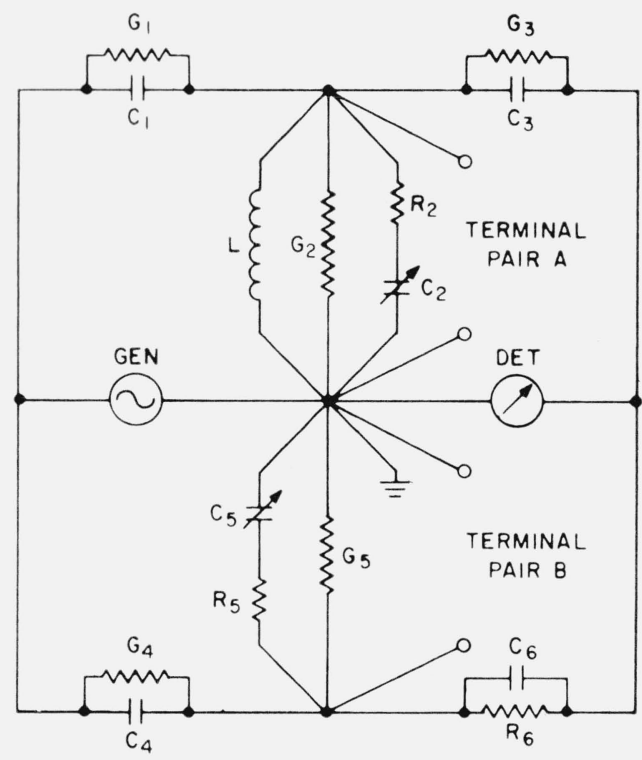

Figure 2. Twin T circuit using impure components.

The circuit of figure 2 is a more realistic representation of the actual circuit. In the figure, the value of each circuit element is taken as its effective value at the frequency at which the instrument is used. If $R_{2}$ is lumped with $G_{2}$ for the present, and $R_{5}$ with $G_{5}$, the null equations for the circuit of figure 2 are

$$
\begin{aligned}
G_{1} G_{3}\left(G_{4}\right. & \left.+G_{5}+\frac{1}{R_{6}}\right)-\omega^{2}\left(C_{1} G_{3}+C_{3} G_{1}\right)\left(C_{4}+C_{5}+C_{6}\right)-\omega^{2} C_{1} C_{3}\left(G_{4}+G_{5}+\frac{1}{R_{6}}\right) \\
& +\frac{G_{4}}{R_{6}}\left(G_{1}+G_{2}+G_{3}\right)-\omega^{2}\left(\frac{C_{4}}{R_{6}}+C_{6} G_{4}\right)\left(C_{1}-\frac{1}{\omega^{2} L}+C_{2}+C_{3}\right)-\omega^{2} C_{4} C_{6}\left(G_{1}+G_{2}+G_{3}\right)=0,
\end{aligned}
$$

and

$$
\begin{aligned}
& G_{1} G_{3}\left(C_{4}+C_{5}+C_{6}\right)+\left(C_{1} G_{3}+C_{3} G_{1}\right)\left(G_{4}+G_{5}+\frac{1}{R_{6}}\right)-\omega^{2} C_{1} C_{3}\left(C_{4}+C_{5}+C_{6}\right) \\
& \quad+\frac{G_{4}}{R_{6}}\left(C_{1}-\frac{1}{\omega^{2} L}+C_{2}+C_{3}\right)+\left(\frac{C_{4}}{R_{6}}+C_{6} G_{4}\right)\left(G_{1}+G_{2}+G_{3}\right)-\omega^{2} C_{4} C_{6}\left(C_{1}-\frac{1}{\omega^{2} L}+C_{2}+C_{3}\right)=0 .
\end{aligned}
$$

In the appendix, these equations are reduced to give the four expressions

$$
\begin{gathered}
G_{x A} \approx \omega^{2}\left(R_{6} \frac{C_{1} C_{3}}{C_{4}}\right) \Delta C_{5}-\omega^{2}\left(R_{4} C_{4}-R_{6} C_{6}\right)\left(\Delta C_{2}+C_{x A}\right)-\Delta G_{2}, \\
G_{x B} \approx \frac{-\Delta C_{2}^{\prime}}{\left(R_{6} \frac{C_{1} C_{3}}{C_{4}}\right)}\left[1-\omega^{2} R_{2}\left(C_{2}+C_{2}^{\prime}\right)\left(R_{4} C_{4}-R_{6} C_{6}\right)\right]-\omega^{2}\left(R_{1} C_{1}+R_{3} C_{3}\right)\left(\Delta C_{5}^{\prime}+C_{x B}\right)-\Delta G_{5}, \\
C_{x A} \approx-\Delta C_{2}\left[1-\omega^{2} R_{2}\left(C_{2}+C_{2}^{\prime}\right)\left(R_{4} C_{4}-R_{6} C_{6}\right)\right]-\left[R_{1} C_{1}+R_{3} C_{3}-R_{4} C_{4}+R_{6} C_{6}+R_{5}\left(C_{5}+C_{5}^{\prime}\right)\right] G_{x A},
\end{gathered}
$$

and

$$
C_{x B} \approx-\Delta C_{5}^{\prime}+\left[R_{1} C_{1}+R_{3} C_{3}-R_{4} C_{4}+R_{6} C_{6}-R_{2}\left(C_{2}+C_{2}^{\prime}\right)\right] G_{x B}
$$

For the instrument whose construction is described later, these equations are accurate to within a few parts in $10^{6}$. If the instrument is to be self-calibrating for conductance 
measurements, eqs (14) and (15) must be reduced to the form

and

$$
G_{x A}+\Delta G_{2}=\omega^{2} P \Delta C_{5},
$$

$$
G_{x B}+\Delta G_{5}=\frac{-\Delta C_{2}^{\prime}}{P}
$$

In order to accomplish this, define

$$
K \equiv R_{1} C_{1}+R_{3} C_{3}-R_{4} C_{4}+R_{6} C_{6} .
$$

Equations (16) and (17) then become

and

$$
C_{x A} \approx-\Delta C_{2}\left[1-\omega^{2} R_{2}\left(C_{2}+C_{2}^{\prime}\right)\left(R_{4} C_{4}-R_{6} C_{6}\right)\right]-\left[K+R_{5}\left(C_{5}+C_{5}^{\prime}\right)\right] G_{x A}
$$

$$
C_{x B} \approx-\Delta C_{5}^{\prime}+\left[K-R_{2}\left(C_{2}+C_{2}^{\prime}\right)\right] G_{x B} .
$$

When measuring a nearly pure conductance, sufficient accuracy is obtained for $C_{x A}$ if the term $\omega^{2} R_{2}\left(C_{2}+C_{2}^{\prime}\right)\left(R_{4} C_{4}-R_{6} C_{6}\right)$ is neglected. Then (19) becomes

$$
C_{x A} \approx-\Delta C_{2}-\left[K+R_{5}\left(C_{5}+C_{5}^{\prime}\right)\right] G_{x A} .
$$

If a nearly pure conductance is measured on terminal-pair $A$, then on terminal-pair $B$, using the same capacitor operated over an identical range for both measurements, then

and

$$
G_{x A}=G_{x B}=G_{x}
$$

$$
R_{5}\left(C_{5}+C_{5}^{\prime}\right)=R_{2}\left(C_{2}+C_{2}^{\prime}\right)=R\left(C+C^{\prime}\right) .
$$

Since $C_{x A}=C_{x B}$,

$$
-\Delta C_{2}-\left[K+R\left(C+C^{\prime}\right)\right] G_{x}=-\Delta C_{5}^{\prime}+\left[K-R\left(C+C^{\prime}\right)\right] G_{x}
$$

from which is obtained

$$
K=\frac{\Delta C_{5}^{\prime}-\Delta C_{2}}{2 G_{x}^{\prime}}
$$

which can be evaluated.

Now, $R_{1} C_{1}, R_{3} C_{3}$, and $R_{4} C_{4}$ are likely to be at least an order of magnitude smaller than $R_{6} C_{6}$. When this is true, $K \approx R_{6} C_{6}$,

and

$$
C_{x A}+\Delta C_{2} \approx-\left[K+R_{5}\left(C_{5}+C_{5}^{\prime}\right)\right] G_{x A},
$$

$$
C_{x B}+\Delta C_{5}^{\prime} \approx\left[K-R_{2}\left(C_{2}+C_{2}^{\prime}\right)\right] G_{x B} .
$$

Equations (14) and (15) can now be rewritten

and

$$
G_{x A} \approx \omega^{2} P \Delta C_{5}-\omega^{2} K\left[K+R_{5}\left(C_{5}+C_{5}^{\prime}\right)\right] G_{x A}-\Delta G_{2}
$$

$$
G_{x B} \approx \frac{-\Delta C_{2}^{\prime}}{P}\left[1+\omega^{2} K R_{2}\left(C_{2}+C_{2}^{\prime}\right)\right]-\omega^{2}\left(R_{1} C_{1}+R_{3} C_{3}\right)\left[K-R_{2}\left(C_{2}+C_{2}^{\prime}\right)\right] G_{x B}-\Delta G_{5}
$$

If the terms $\omega^{2} K\left[K+R_{5}\left(C_{5}+C_{5}^{\prime}\right)\right], \omega^{2} K R_{2}\left(C_{2}+C_{2}^{\prime}\right)$, and $\omega^{2}\left(R_{1} C_{1}+R_{3} C_{3}\right)\left[K-R_{2}\left(C_{2}+C_{2}^{\prime}\right)\right]$ are insignificant compared to unity, the expressions for the conductance reduce to the desired form.

If $\Delta G_{2}=\Delta G_{5}$, or if both are insignificant compared to $G_{x}, P$ can be determined as it was in the ideal case. It is possible to insure that $\Delta C_{2}=\Delta C_{5}$, and that $\Delta G_{2}=\Delta G_{5}$ in the two measure- 
ments, by using the same variable capacitors operated over an identical range to measure $G_{x}$ and $C_{x}$ in both cases. This requires both $G_{x}$ and the capacitors which measure $G_{x}$ and $C_{x}$ to be switched from terminal to terminal.

It is possible to adjust $P$ to within the limits of stability and resettability of the capacitor used to measure $G_{x}$. In a good two-terminal air capacitor equipped with a precision coaxial connector, the error is no more than a few parts in $10^{5}$ of the maximum capacitance. Since changes in effective capacitance are not known to better than several parts in $10^{4}$ at present, adjusting $P$ in this way allows one to measure conductance to the same accuracy as that to which a change in effective capacitance is known.

With the constants of the instrument known, it is only necessary to evaluate the residual impedances associated with the external variable capacitors, perhaps by the method of Field and Sinclair [5], in order to make quite good measurements of either conductance or reactance.

\section{Construction of the Instrument}

In converting the twin $T$ circuit to a working instrument, several facts must be considered. Since it is necessary to move the standard capacitors from one pair of terminals to the other when evaluating the constants of the instrument, the standard capacitors cannot be made an integral part of the instrument. If the capacitors are not to be built in, more than one pair of terminals must be provided to allow paralleling the unknown admittance and the capacitor which measures $C_{x}$. Multiple terminals are also desirable because they simplify calibration of the variable capacitors used with the instrument. If multiple terminals are to be used, it is desirable for each pair to connect to a common point, the "bridge corner," and for the conductors to be mutually perpendicular in order to reduce mutual impedances as much as possible. The fact that it is possible to connect external variable capacitors of different ranges for $C_{2}$ and $C_{5}$ requires changing the values of $L$ and $G_{2}$ necessary to provide initial balance. Since multiple terminals are already necessary, it is just as well to provide for the attachment of external standards for $L$ and $G_{2}$. As a result, the actual instrument becomes very simple. It is only necessary to provide for mounting the four fixed elements, the series elements of the $T$, in a base to which are attached the two sets of multiple terminals.

As actually constructed, the instrument takes the form shown in figures 3,5 , and 6 . The four series elements are mounted in holes bored parallel to the four sides of a 2 in. by 6 in. brass block. The holes are extended through the edges of the block to provide access to the components from one end, and to form the outer conductor of a short section of $3 / 4 \mathrm{in}$. coaxial line on the other. Holes bored through diagonally opposite corners from face to face provide the outer conductors for two more short sections of line. When center conductors are added, six short sections of coaxial line, whose center conductors meet in a point, are formed at two diagonally opposite corners of the block. Two of these line sections connect to the series components, and the other four are equipped with NBS-Woods [6] precision coaxial connectors to provide the bridge terminals. The junction point of the six line sections forms the "bridge corner."

Capacitors $C_{1}$ and $C_{3}$ are coaxial capacitors with nominal values of $10 \mathrm{pF} . \quad C_{4}$ is a parallel plate capacitor whose nominal value is $1 \mathrm{pF} . \quad R_{6}$ is a commercial metal film resistor whose value is selected to give the proper value of $P$ at the frequency at which the instrument is to be used.

Since it is necessary to adjust $P$, one or more of the series elements must be adjustable. $C_{4}$ is provided with two adjustments. Each plate has one half its surface cut about $1 / 32$ in. lower than the other half, so that rotating one plate with respect to the other causes a change of about 10 percent in its capacitance. Final adjustment of $P$ is accomplished by means of a trimming screw which extends into the space between the two plates. Gross changes in $P$ can be accomplished by replacing the resistor $R_{6}$ with another of different value. 


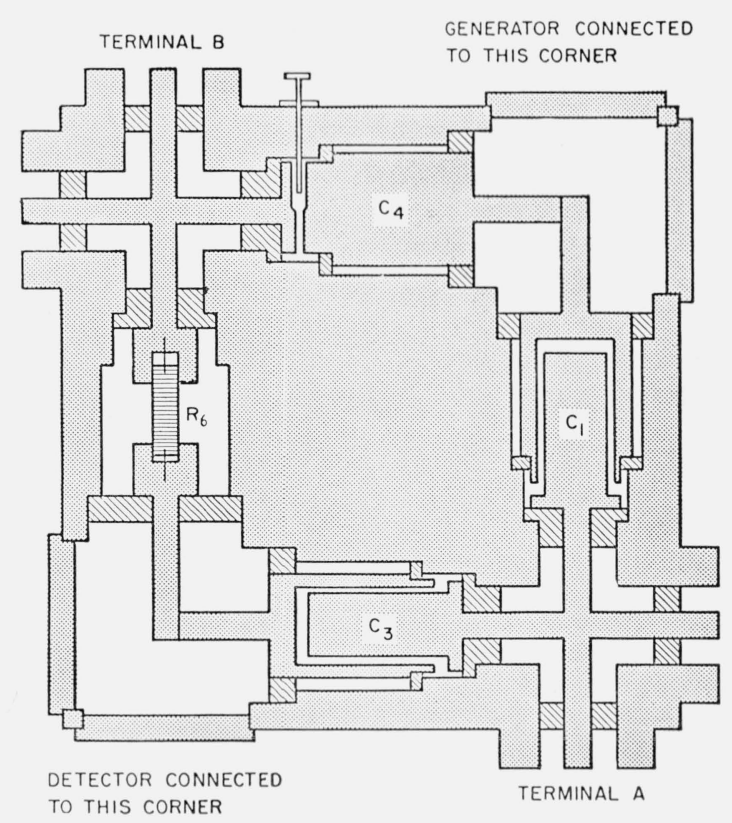

Figure 3. Cross section through the brass block showing construction of the instrument.

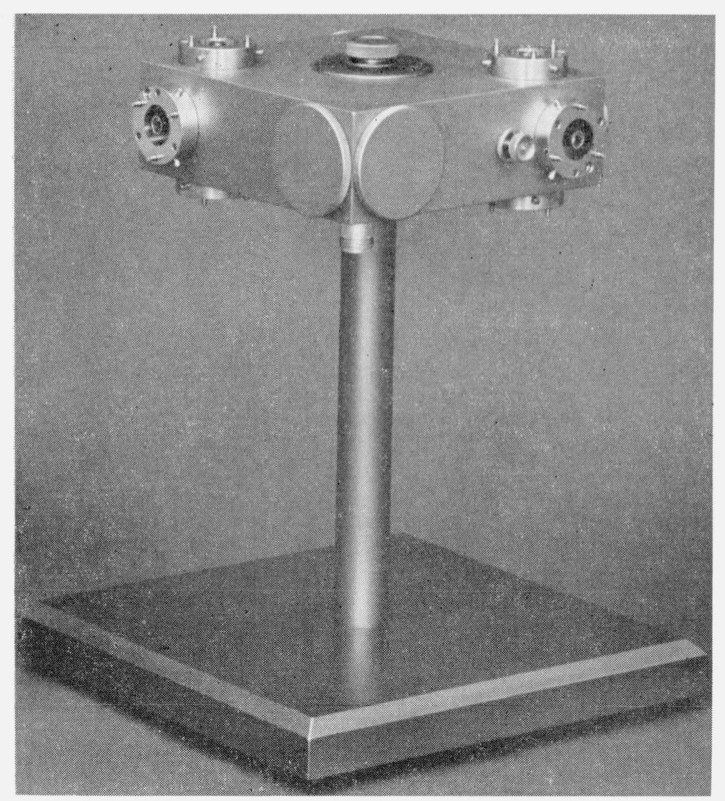

FiguRE 5. The basic instrument showing connector details and $\mathrm{C}_{4}$ trimmer.

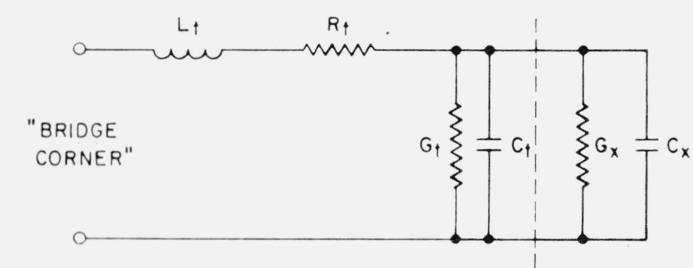

Figure 4. Equivalent circuit used in calculating the effect of terminal impedances on measured $\mathrm{Y}_{\mathbf{x}}$.

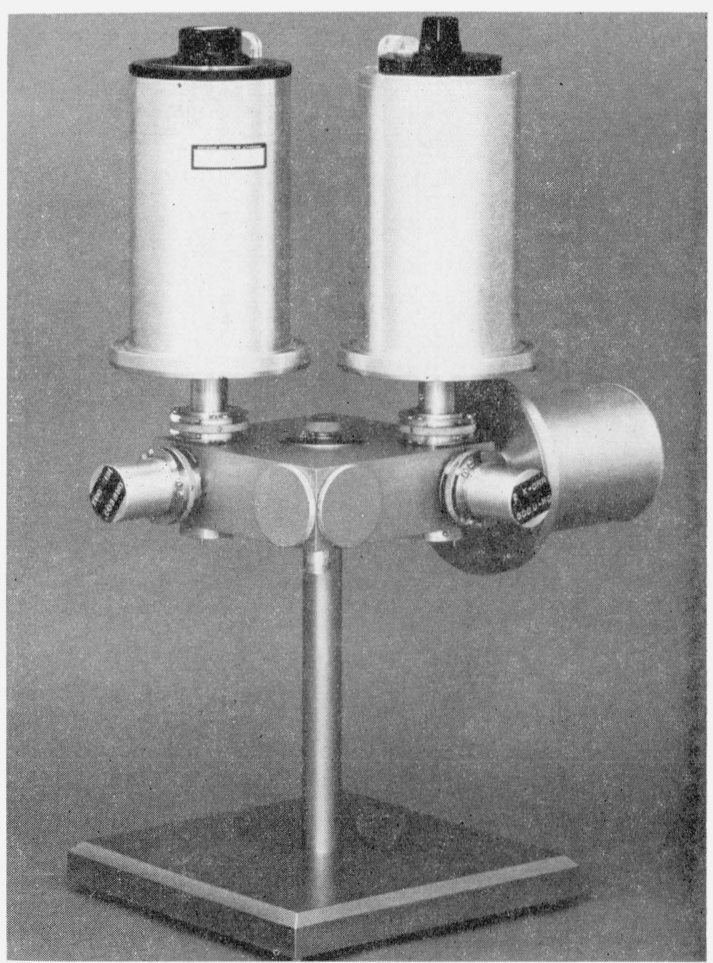

Figure 6. The basic instrument with standards attached for measuring a 200-ohm resistor.

\section{Experimental Results}

$P$ was adjusted to equal $\frac{1}{\omega}$, at $\omega=10^{7}$ radians/sec, by measuring a $1000 \Omega$ resistor using a 10 to $110 \mathrm{pF}$ variable standard capacitor. This resistor, as were those used later, is a high quality commercial metal-film resistor mounted in a shielding can which is equipped with a NBS-Woods Precision Coaxial Connector. The three standard capacitors used are equipped with similar connectors. 
When $P$ had been adjusted to the point that the capacitance change on one terminal was within a few parts in $10^{5}$ of that on the other, over an identical capacitance range, $P$ was considered to be adjusted to within a few parts in $10^{5}$. The adjustment was checked by measuring a $2000 \Omega$ resistor using the same capacitor, a $20,000 \Omega$ resistor using a 7 to $17 \mathrm{pF}$ capacitor, and 100,200 , and $500 \Omega$ resistors using a 100 to $1100 \mathrm{pF}$ capacitor. The results are summarized in table 1 . Since the error in resetting the capacitors is a few parts in $10^{5}$ of their maximum capacitance, the adjustment of $P$ is probably correct to within a few parts in $10^{5}$.

With the values obtained for $\Delta C_{5}$, or $\Delta C_{2}{ }^{\prime}$, in checking the adjustment of $P$, and knowing that $P$ is $10^{-7} \pm$ a few parts in $10^{5}$, it is possible to calculate the effective conductance of each of the resistors at $10^{7}$ radians/sec. The results of the calculations appear in table 2. The measurements of $C_{x}$ obtained in checking the adjustment of $P$ may be used to calculate a value for $K$. The results of the calculations appear in table 3 .

TABLE 1. Verification of the adjustment of $\mathrm{P}$

\begin{tabular}{|c|c|c|c|c|}
\hline $\begin{array}{l}\text { Resistors } \\
\text { measured }\end{array}$ & $\begin{array}{c}\text { Terminal } \\
\text { used }\end{array}$ & $\begin{array}{l}\text { Capacitor } \\
\text { used }\end{array}$ & $\Delta C$ & $\begin{array}{l}\% \text { Diff. } \\
=\left(\frac{\Delta C_{2}}{\Delta C_{2}}-1\right) 100\end{array}$ \\
\hline $\begin{array}{l}\Omega \\
100 \\
100\end{array}$ & $\underset{B}{A}$ & $\begin{array}{c}p F \\
100-1100 \\
100-1100\end{array}$ & $\begin{array}{c}p F \\
999.40 \\
999.37\end{array}$ & +0.003 \\
\hline $\begin{array}{l}200 \\
200\end{array}$ & $\begin{array}{l}A \\
B\end{array}$ & $\begin{array}{l}100-1100 \\
100-1100\end{array}$ & $\begin{array}{l}499.00 \\
498.94\end{array}$ & +.012 \\
\hline $\begin{array}{l}500 \\
500\end{array}$ & $\begin{array}{l}A \\
B\end{array}$ & $\begin{array}{l}100-1100 \\
100-1100\end{array}$ & $\begin{array}{l}192.92 \\
192.92\end{array}$ & .000 \\
\hline $\begin{array}{l}1000 \\
1000\end{array}$ & $\stackrel{A}{B}$ & $\begin{array}{l}10-110 \\
10-110\end{array}$ & $\begin{array}{l}100.050 \\
100.051\end{array}$ & -.001 \\
\hline $\begin{array}{l}2000 \\
2000\end{array}$ & $\stackrel{A}{B}$ & $\begin{array}{l}10-110 \\
10-110\end{array}$ & $\begin{array}{l}50.206 \\
50.208\end{array}$ & -.004 \\
\hline $\begin{array}{l}20,000 \\
20,000 \\
20,000\end{array}$ & $\begin{array}{l}A \\
B \\
A\end{array}$ & $\begin{array}{l}7-17 \\
7-17 \\
7-17\end{array}$ & $\begin{array}{l}\text { 4. } 9994 \\
\text { 4. } 9991 \\
\text { 4. } 9976\end{array}$ & $\begin{array}{l}+.006 \\
-.030\end{array}$ \\
\hline
\end{tabular}

Percentage error in $P$ is $1 / 2 \times$ ( $\%$ diff.).
TABLE 2. Comparison of measured resistance to $d-c$ resistance

\begin{tabular}{|c|c|c|c|c|}
\hline$\Delta C$ & $G=\omega \Delta C$ & $R_{f}=\frac{1}{G}$ & $R_{d c}$ & $\begin{array}{l}\% \text { diff. } \\
=\left(\frac{R_{f}}{R_{d c}}-1\right) 100\end{array}$ \\
\hline$p F$ & Mho & & & \\
\hline 1000.44 & 1. $00044 \times 10^{-2}$ & 99. 956 & 99. 971 & -0.015 \\
\hline $\begin{array}{l}499.71 \\
199.84\end{array}$ & $1000-2$ & & & -.018 \\
\hline $\begin{array}{l}199.84 \\
100.031\end{array}$ & $\begin{array}{l}\text { 1. } 9984 \times 10^{-3} \\
\text { 1. } 00031 \times 10^{-3}\end{array}$ & $\begin{array}{l}500.40 \\
999.69\end{array}$ & $\begin{array}{l}500.428 \\
999.853\end{array}$ & $\begin{array}{l}-.006 \\
-.016\end{array}$ \\
\hline 50.02 & 5. $0029 \times 10^{-4}$ & 1998.8 & 1999. 52 & -.036 \\
\hline 80 & 5. $0180 \times 10^{-5}$ & 19928 & 19998.4 & 3 \\
\hline
\end{tabular}

TABLE 3. Calculation of $K$ from measured capacitance

\begin{tabular}{c|c|c|c}
\hline \hline$G_{x}$ & $\Delta C^{\prime}{ }_{5}$ & $\Delta C_{2}$ & \multirow{2}{*}{$K=\frac{\Delta C^{\prime}{ }_{5}-\Delta C_{2}}{2 G_{x}}$} \\
\cline { 1 - 2 } & & & \\
\cline { 1 - 2 } Mho & $p F$ & & \\
0.01 & 3.503 & -5.942 & $4.72 \times 10^{-10}$ \\
.005 & -0.125 & -4.821 & $4.70 \times 10^{-10}$ \\
.002 & -2.440 & -4.308 & $4.67 \times 10^{-10}$ \\
.001 & -2.3145 & -3.2559 & $4.71 \times 10^{-10}$ \\
.0005 & -2.4132 & -2.8826 & $4.69 \times 10^{-10}$ \\
\hline
\end{tabular}

The admittance measured by the instrument is the effective admittance at the "bridge corners." In order to calculate $Y_{x}$ accurately, it is necessary to know the effect of the residual impedances between the terminal and the "bridge corner." The equivalent circuit of figure 4 is a reasonable representation of the residual impedances associated with the terminal.

When the instrument is nulled initially with the terminal open, the effective admittance at the "bridge corner" is given by

$$
Y_{e}=\frac{G_{t}\left(1+R_{t} G_{t}\right)+\omega^{2} R_{t} C_{t}^{2}+j \omega\left[C_{t}-L_{t} G_{t}^{2}-\omega^{2} L_{t} C_{t}^{2}\right]}{\left(1+R_{t} G_{t}-\omega^{2} L_{t} C_{t}\right)^{2}+\omega^{2}\left(R_{t} C_{t}+L_{t} G_{t}\right)^{2}} .
$$

For the purpose of estimating the magnitudes of the residual impedances, the terminal can be considered to be $1 \frac{3 / 8}{\mathrm{in}}$. of $50 \Omega, 3 / 4 \mathrm{in}$, brass air line. Since the series resistance and inductance in similar sections of line have been determined to be 0.8 milliohm/in. and $4.4 \times 10^{-9} \mathrm{H} / \mathrm{in}$. at $10^{7}$ radians/sec, $R_{t}$ is about 1.1 milliohms, and $L_{t}$ about $6.2 \times 10^{-9} \mathrm{H} . \quad C_{t}$ is about $2.5 \mathrm{pF}$, and $G_{t}$ is less than $1 \mu$ mho. Using these values for the residual impedances, the equation can be reduced to

$$
Y_{e} \approx G_{t}+j \omega C_{t}, \text { at } \omega=10^{7} \text { radians } / \mathrm{sec} .
$$

When an unknown admittance, $Y_{x}=G_{x}+j \omega C_{x}$, is connected to the terminal, the effective admittance at the "bridge corner" is given by

$$
Y_{e}^{\prime}=\frac{\left(G_{t}+G_{x}\right)\left[1+R_{t}\left(G_{t}+G_{x}\right)\right]+\omega^{2} R_{t}\left(C_{t}+C_{x}\right)^{2}+j \omega\left[C_{t}+C_{x}-L_{t}\left(G_{t}+G_{x}\right)^{2}-\omega^{2} L_{t}\left(C_{t}+C_{x}\right)^{2}\right]}{\left[1+R_{t}\left(G_{t}+G_{x}\right)-\omega^{2} L_{t}\left(C_{t}+C_{x}\right)\right]^{2}+\omega^{2}\left[R_{t}\left(C_{t}+C_{x}\right)+L_{t}\left(G_{t}+G_{x}\right)\right]^{2}} .
$$


For $G_{x} \leq 10^{-2}$ and $C_{x}<10^{-11}$, this reduces to

and

$$
Y_{e}^{\prime} \approx G_{t}+G_{x}+j \omega\left(C_{t}+C_{x}-L_{t} G_{x}^{2}\right)
$$

$$
\Delta Y_{e}=Y_{e}^{\prime}-Y_{e} \approx G_{x}+j \omega\left(C_{x}-L_{t} G_{x}^{2}\right) .
$$

The error in the measured conductance is insignificant, and the measured capacitance is in error by the term $\left(-L_{t} G_{x}{ }^{2}\right)$. Before the reactance associated with a nearly pure conductance can be accurately measured, it is necessary to evaluate this inductance, as well as the series resistance associated with the capacitor used to measure the conductance. It should be mentioned that these residual impedances cause no error in evaluating the constants of the instrument if care is taken to make all the terminals as nearly identical as possible.

\section{Discussion}

The measurements of $P$, which are summarized in table 1 , indicate that it is possible to adjust $P$ to within a few parts in $10^{5}$ of $1 / \omega$. The limit on the accuracy of adjustment of $P$ is the limit of stability and resettability of the standard capacitors used in making the adjustment.

Comparison of resistance measured at $10^{7}$ radians/sec with that measured at zero frequency (table 2) indicates that the instrument is capable of measuring resistance to within a few hundredths of a percent. The very close agreement between rf and d-c values must be considered fortuitous at present because changes in capacitance are not known to much better than \pm 0.05 percent.

The values calculated for $K$ (table 3 ) have a scatter of less than 1 percent of $K$. This indicates that it will be possible to make quite good measurements of the reactance associated with a nearly pure conductance. Apparently, the accuracy of such measurements will be limited by the accuracy to which the series resistance associated with the standard capacitors and the series inductance associated with the terminal can be determined.

While the instrument has been proved only at a frequency of $10^{7}$ radians/sec, there is no apparent reason why the same principle can not be used at much higher, or at much lower, frequencies. The main deinciency of the instrument lies in the range of conductance which can be measured accurately. At present the range is limited, by the relationship $G_{x}=\omega \Delta C$, to two or three decades of conductance by the range of capacitance changes which is easily obtainable. Since the magnitude of the conductance measurable by a given capacitor depends on the frequency, the range of conductances measurable by the instrument is frequency dependent. The deficiency need not be too serious if it proves feasible to use the instrument to measure conductances for use as standards when calibrating other instruments capable of covering a greater conductance range.

The instrument has demonstrated its ability to make conductance measurements limited in accuracy only by the accuracy to which changes in capacitance are known. This being the case, it is possible to immediately realize an order of magnitude increase in the accuracy of those conductance measurements which are within the range of the instrument. Of greater significance is the fact that the instrument demonstrates a principle which makes it possible to refer resistance measurements directly to standards of capacitance, thus placing the meastrement of resistance and reactance on a common basis.

\section{Appendix}

The null equations for the circuit of figure 2 are

$$
\begin{aligned}
G_{1} G_{3}\left(G_{4}\right. & \left.+G_{5}+\frac{1}{R_{6}}\right)-\omega^{2}\left(C_{1} G_{3}+C_{3} G_{1}\right)\left(C_{4}+C_{5}+C_{6}\right)-\omega^{2} C_{1} C_{3}\left(G_{4}+G_{5}+\frac{1}{R_{6}}\right) \\
& +\frac{G_{4}}{R_{6}}\left(G_{1}+G_{2}+G_{3}\right)-\omega^{2}\left(\frac{C_{4}}{R_{6}}+C_{6} G_{4}\right)\left(C_{1}-\frac{1}{\omega^{2} L}+C_{2}+C_{3}\right)-\omega^{2} C_{4} C_{6}\left(G_{1}+G_{2}+G_{3}\right)=0,
\end{aligned}
$$


and

$$
\begin{aligned}
& G_{1} G_{3}\left(C_{4}+C_{5}+C_{6}\right)+\left(C_{1} G_{3}+C_{3} G_{1}\right)\left(G_{4}+G_{5}+\frac{1}{R_{6}}\right)-\omega^{2} C_{1} C_{3}\left(C_{4}+C_{5}+C_{6}\right) \\
& \quad+\frac{G_{4}}{R_{6}}\left(C_{1}-\frac{1}{\omega^{2} L}+C_{2}+C_{3}\right)+\left(\frac{C_{4}}{R_{6}}+C_{6} G_{4}\right)\left(G_{1}+G_{2}+G_{3}\right)-\omega^{2} C_{4} C_{6}\left(C_{1}-\frac{1}{\omega^{2} L}+C_{2}+C_{3}\right)=0 .
\end{aligned}
$$

A measurement can be made by connecting an unknown admittance, $Y_{x}=G_{x}+j \omega C_{x}$, either to terminal-pair $A$, or to terminal-pair $B$. When $Y_{x}$ is connected to terminal-pair $A$, $C_{2}$ must be replaced by $C_{2}^{\prime}+C_{x}, G_{2}$ by $G_{2}^{\prime}+G_{x}, G_{5}$ by $G_{5}^{\prime}$, and $C_{5}$ by $C_{5}^{\prime}$ in the null equations. Similar changes must be made when the unknown admittance is connected to terminal-pair $B$. If $\Delta C_{2}=C_{2}^{\prime}-C_{2}, \Delta G_{2}=G_{2}^{\prime}-G_{2}, \Delta C_{5}=C_{5}^{\prime}-C_{5}$, and $\Delta G_{5}=G_{5}^{\prime}-G_{5}$, and subscripts $A$ and $B$ denote the terminal-pair to which the unknown admittance is connected, subtracting the initial null equations from the final ones yields the four expressions

$$
\begin{aligned}
C_{x A}\left[1+\frac{R_{6} G_{4} C_{6}}{C_{4}}\right]=-\left[1+\frac{R_{6} G_{4} C_{6}}{C_{4}}\right] \Delta C_{2}+ & {\left[\frac{R_{6} G_{1} G_{3}}{\omega^{2} C_{4}}-\frac{R_{6}}{C_{4}} C_{1} C_{3}\right] \Delta G_{5} } \\
& +\left[\frac{G_{4}}{\omega^{2} C_{4}}-R_{6} C_{6}\right]\left(\Delta G_{2}+G_{x}\right)-\frac{R_{6}}{C_{4}}\left(C_{1} G_{3}+C_{3} G_{1}\right) \Delta C_{5}, \\
C_{x B}\left[\frac{\omega^{2} R_{6} C_{1} C_{3}}{C_{4}}-\frac{R_{6} G_{1} G_{3}}{C_{4}}\right]=-\left[\frac{\omega^{2} R_{6} C_{1} C_{3}}{C_{4}}-\frac{R_{6} G_{1} G_{3}}{C_{4}}\right] \Delta C_{5}+\left[1+\frac{R_{6} G_{4} C_{6}}{C_{4}}\right] \Delta G_{2} & +\frac{R_{6}}{C_{4}}\left(C_{1} G_{3}+C_{3} G_{1}\right)\left(\Delta G_{5}+G_{x}\right)+\left(\frac{G_{4}}{C_{4}}-\omega^{2} R_{6} C_{6}\right) \Delta C_{2}, \\
G_{x A}\left[1+\frac{R_{6} G_{4} C_{6}}{C_{4}}\right]=\left[\frac{\omega^{2} R_{6} C_{1} C_{3}}{C_{4}}-\frac{R_{6} G_{1} G_{3}}{C_{4}}\right] \Delta C_{5}+\left[\omega^{2} R_{6} C_{6}-\frac{G_{4}}{C_{4}}\right]\left(\Delta C_{2}+C_{x}\right) & -\frac{R_{6}}{C_{4}}\left(C_{1} G_{3}+C_{3} G_{1}\right) \Delta G_{5}-\left(1+\frac{R_{6} G_{4} C_{6}}{C_{4}}\right) \Delta G_{2},
\end{aligned}
$$

and

$$
\begin{aligned}
G_{x B}\left[\frac{R_{6} C_{1} C_{3}}{C_{4}}-\frac{R_{6} G_{1} G_{3}}{\omega^{2} C_{4}}\right]=-\left(1+\frac{R_{6} G_{4} C_{6}}{C_{4}}\right) \Delta & C_{2}-\frac{R_{6}}{C_{4}}\left(C_{1} G_{3}+C_{3} G_{1}\right)\left(\Delta C_{5}+C_{x}\right) \\
& +\left[\frac{R_{6} G_{1} G_{3}}{\omega^{2} C_{4}}-\frac{R_{6} C_{1} C_{3}}{C_{4}}\right] \Delta G_{5}+\left[\frac{G_{4}}{\omega^{2} C_{4}}-R_{6} C_{6}\right] \Delta G_{2} .
\end{aligned}
$$

If $C_{1}, C_{3}$, and $C_{4}$ are good three-terminal capacitors, their losses will be due entirely to series resistance, in which case,

and

$$
G_{1} \approx \omega^{2} R_{1} C_{1}^{2}, G_{3} \approx \omega^{2} R_{3} C_{3}^{2}
$$

$$
G_{4} \approx \omega^{2} R_{4} C_{4}^{2} .
$$

If $C_{2}$ and $C_{5}$ are good two-terminal capacitors, changes in their conductance will be due entirely to series resistance, and

$$
\begin{aligned}
& \Delta G_{2} \approx \omega^{2} R_{2}\left(C_{2}^{\prime 2}-C_{2}^{2}\right)=\omega^{2} R_{2}\left(C_{2}+C_{2}^{\prime}\right) \Delta C_{2}, \\
& \Delta G_{5} \approx \omega^{2} R_{5}\left(C_{5}+C_{5}^{\prime}\right) \Delta C_{5} .
\end{aligned}
$$

In the instrument described,

$$
\begin{aligned}
& C_{1} \approx C_{3} \approx 10 \mathrm{pF} \\
& C_{4} \approx 1 \mathrm{pF}
\end{aligned}
$$




$$
\begin{aligned}
& C_{2} \leq 1000 \mathrm{pF} \\
& C_{5} \leq 1000 \mathrm{pF} \\
& R_{6} \approx 1000 \Omega \text { at } 10^{7} \mathrm{radians} / \mathrm{sec} \\
& R_{6} \approx 100 \Omega \text { at } 10^{8} \mathrm{radians} / \mathrm{sec} .
\end{aligned}
$$

and

It is not unreasonable to assume $R_{1} \approx R_{2} \approx R_{3} \approx R_{4} \approx R_{5}<0.1 \Omega$ and

$$
C_{6} \approx 1 \mathrm{pF} \text {. }
$$

The null equations can then be reduced by using the following approximations:

$$
\begin{gathered}
{\left[1+\frac{G_{4} R_{6} C_{6}}{C_{4}}\right] \approx\left[1+\frac{\omega^{2} R_{4} C_{4}^{2} R_{6} C_{6}}{C_{4}}\right] \approx 1+\omega^{2} R_{4} R_{6} C_{4} C_{6} \approx 1,} \\
{\left[\frac{R_{6} G_{1} G_{3}}{\omega^{2} C_{4}}-\frac{R_{6} C_{1} C_{3}}{C_{4}}\right] \approx \frac{R_{6} C_{1} C_{3}}{C_{4}}\left[\omega^{2} R_{1} R_{3} C_{1} C_{3}-1\right] \approx-\frac{R_{6} C_{1} C_{3},}{C_{4}},} \\
{\left[\frac{\omega^{2} R_{6} C_{1} C_{3}}{C_{4}}-\frac{R_{6} G_{1} G_{3}}{C_{4}}\right]=\omega^{2}\left[\frac{R_{6} C_{1} C_{3}}{C_{4}}-\frac{R_{6} G_{1} G_{3}}{\omega^{2} C_{4}}\right] \approx \omega^{2} \frac{R_{6} C_{1} C_{3}}{C_{4}},} \\
{\left[\frac{G_{4}}{\omega^{2} C_{4}}-R_{6} C_{6}\right] \approx\left[\frac{\omega^{2} R_{4} C_{4}^{2}}{\omega^{2} C_{4}}-R_{6} C_{6}\right] \approx R_{4} C_{4}-R_{6} C_{6},} \\
{\left[\frac{G_{4}}{C_{4}}-\omega^{2} R_{6} C_{6}\right]=\omega^{2}\left[\frac{G_{4}}{\omega^{2} C_{4}}-R_{6} C_{6}\right] \approx \omega^{2}\left[R_{4} C_{4}-R_{6} C_{6}\right],} \\
{\left[C_{1} G_{3}+C_{3} G_{1}\right] \approx\left[\omega^{2} R_{3} C_{3}^{2} C_{1}+\omega^{2} R_{1} C_{1}^{2} C_{3}\right] \approx \omega^{2} C_{1} C_{3}\left(R_{1} C_{1}+R_{3} C_{3}\right) .}
\end{gathered}
$$

Using expressions (a), (b), (d), and (f), eq (A3) is reduced to

$$
C_{x A} \approx-\Delta C_{2}-\frac{R_{6} C_{1} C_{3}}{C_{4}} \Delta G_{5}+\left(R_{4} C_{4}-R_{6} C_{6}\right)\left(\Delta G_{2}+G_{x A}\right)-\frac{\omega^{2} R_{6} C_{1} C_{3}}{C_{4}}\left(R_{1} C_{1}+R_{3} C_{3}\right) \Delta C_{5} .
$$

Since

and

$$
\begin{aligned}
& \Delta G_{5} \approx \omega^{2} R_{5}\left(C_{5}+C_{5}^{\prime}\right) \Delta C_{5}, \\
& \Delta G_{2} \approx \omega^{2} R_{2}\left(C_{2}+C_{2}^{\prime}\right) \Delta C_{2},
\end{aligned}
$$

$$
\begin{gathered}
\omega^{2} R_{6} \frac{C_{1} C_{3}}{C_{4}} \Delta C_{5} \approx G_{x A}, \\
C_{x A} \approx-\Delta C_{2}\left[1-\omega^{2} R_{2}\left(C_{2}+C_{2}^{\prime}\right)\left(R_{4} C_{4}-R_{5} C_{6}\right)\right]-\left[R_{1} C_{1}+R_{3} C_{3}-R_{4} C_{4}+R_{6} C_{6}+R_{5}\left(C_{5}+C_{5}^{\prime}\right)\right] G_{x A},
\end{gathered}
$$

which is eq (16).

Using (a), (c), (e), and (f), eq (A4) is reduced to

$$
C_{x B} \approx-\Delta C_{5}+\left(R_{1} C_{1}+R_{3} C_{3}\right)\left(\Delta G_{5}+G_{x B}\right)+\frac{\Delta C_{2}\left(R_{4} C_{4}-R_{6} C_{6}\right)}{\left(R_{6} \frac{C_{1} C_{3}}{C_{4}}\right)}+\frac{\Delta G_{2}}{\omega^{2}\left(R_{6} \frac{C_{1} C_{3}}{C_{4}}\right)} \cdot
$$

Since

and

$$
\frac{\Delta C_{2}}{R_{6} \frac{C_{1} C_{3}}{C_{4}}} \approx-G_{x B}
$$

$$
\omega^{2}\left(R_{1} C_{1}+R_{3} C_{3}\right) R_{5}\left(C_{5}+C_{5}^{\prime}\right)<<1
$$


this can be further reduced to

which is eq (17).

$$
C_{x B} \approx-\Delta C_{5}+\left[R_{1} C_{1}+R_{3} C_{3}-R_{4} C_{4}+R_{6} C_{6}-R_{2}\left(C_{2}+C_{2}^{\prime}\right)\right] G_{x B}
$$

Using (a), (c), (e), and (f), eq (A5) is reduced to

$$
G_{x A} \approx \frac{\omega^{2} R_{6} C_{1} C_{3}}{C_{4}} \Delta C_{5}-\omega^{2}\left(R_{4} C_{4}-R_{6} C_{6}\right)\left(\Delta C_{2}+C_{x A}\right) \frac{-\omega^{2} R_{6} C_{1} C_{3}}{C_{4}}\left(R_{1} C_{1}+R_{3} C_{3}\right) \Delta G_{5}-\Delta G_{2} .
$$

Since

and

this reduces to

$$
\Delta G_{5} \approx \omega^{2} R_{5}\left(C_{5}+C_{5}^{\prime}\right) \Delta C_{5}
$$

$$
\omega^{2}\left(R_{1} C_{1}+R_{3} C_{3}\right) R_{5}\left(C_{5}+C_{5}^{\prime}\right)<<1,
$$

$$
G_{x A} \approx \frac{\omega^{2} R_{6} C_{1} C_{3}}{C_{4}} \Delta C_{5}-\omega^{2}\left(R_{4} C_{4}-R_{6} C_{6}\right)\left(\Delta C_{2}+C_{x A}\right)-\Delta G_{2}
$$

which is eq (14).

Using expressions (a), (b), (d), and (f), eq (A6) is reduced to

$$
G_{x B} \approx \frac{-\Delta C_{2}}{R_{6} \frac{C_{1} C_{3}}{C_{4}}}-\omega^{2}\left(R_{1} C_{1}+R_{3} C_{3}\right)\left(\Delta C_{5}+C_{x B}\right)-\Delta G_{5}+\frac{\left(R_{4} C_{4}-R_{6} C_{6}\right) \Delta G_{2}}{R_{6} \frac{C_{1} C_{3}}{C_{4}}} .
$$

Since

$$
\begin{gathered}
\Delta G_{2} \approx \omega^{2} R_{2}\left(C_{2}+C_{2}^{\prime}\right) \Delta C_{2}, \\
G_{x B} \approx-\frac{\Delta C_{2}}{R_{6} \frac{C_{1} C_{3}}{C_{4}}}\left[1-\omega^{2} R_{2}\left(C_{2}+C_{2}^{\prime}\right)\left(R_{4} C_{4}-R_{6} C_{6}\right)\right]-\omega^{2}\left(R_{1} C_{1}+R_{3} C_{3}\right)\left(\Delta C_{5}+C_{x B}\right)-\Delta G_{5},
\end{gathered}
$$

eq (15). Equations (A7), (A8), (A9), and (A10) should be accurate to within a few parts in $10^{6}$ at frequencies up to $10^{8}$ radians/sec for the instrument described.

\section{References}

[1] Powell, R. C., Jickling, R. M., and Hess, A. E., High frequency impedance standards at the National Bureau of Standards. IRE Trans. Instr. I-y, Nos. 3 \& 4, 270 (Dec. 1958).

[2] Tuttle, W. N., Bridged-T and parallel-T null circuits for measurements at radio frequencies, Proc. IRE 28, 23 (Jan. 1940).

[3] Sinclair, D. B., The Twin T-A new type of null instrument for measuring impedance at frequencies up to 30 megacycles. Proc. IRE 28, 310 (July 1940).

[4] Woods, D., A precision V.H.F. twin-T dual admittance bridge and associated standards, Report No. A.I.D. Rad. Elec. 17 (Jan. 1950).

[5] Field, R. F., and Sinclair, D. B., A method for determining the residual inductance and resistance of a variable air condenser at radio frequencies, Proc. IRE, 24, No. 2, 255-274 (Feb. 1936).

[6] Woods, D., A coaxial connector system for precision R.F. measuring instruments and standards. Proc. IEE, 108B, Paper No. 3499E, pp. 205-213 (Mar. 1961). 